\title{
1978 MEMBERSHIP DIRECTORY
}

(Corrected to November 1, 1980)

Permission for Commercial Use Must Be Obtained From the NALLD Executive Secretary

ABEYTA, J., 5248 South 1128 Rd., Alamosa, CO 81101

ABRAMS, W., Education Dept., Capitol Complex, Carson City, NV 89710

ACADIA UNIV., Vaughan Memorial Library, Wolfville BOP IXO, NS Canada

ADAMS, S., Spanish-Portugues, Baylor University-Box 159, Waco, TX 77004

AIKENS, H., Part-Time Studies-Extension, Dalhousie University 3/5, NS, Canada

ADFL BULLETIN-EDITOR, 62 Fifth Avenue, New York, NY 10011

ADV. MGR-HISPANIA, Glenbrook South HS, 4000 W. Lake Ave., Glenview, IL 60025

ALLEN, W., Language Lab, Saint Olaf College, Northfield, MN 55057

ALLEY, G., Dept of State, Foreign Service Inst., Key Blvd., Arlington, VA 22209

ALTMAN, H., Modern Languages, U of Louisville, Louisville, KY 40292

ALTMAN, L., Languages Lab-Hill 313, Sanfrancisco State, San Francisco, CA 94132

AMADA, K., 16-6 Shiomigaoka-Cho, Chiba-Shi 280, Japan

ANASTOS, P., 155 E. 33 St., New York, NY 10016

ANDRASICK, E., Language Lab, Adelphi University, Garden City, NY 11530

ANDREW, A. MC., Language Ctr., University of Sydney, Sydney NSW 2006, Australia APPALACHIAN STATE UNIVERSITY, Lib-Serials, Boone, NC 28608

ARIZONA STATE UNIV., Library-Periodicals, Tempe, AZ 85281

ASSOC. EDUC. COMM. \& TECH., Adv. Manager, 120116 St. NW, Washington, DC 20036

AUCOIN, G., 34 Langley Ave., Dartmouth B2W 2Y6, NS, Canada

AUCEROT, J., Language Learning Ctr., $U$ of Washington, Seattle, WA 98195

AUSTRALIAN NAT UNIV., Gen. Studies Library, PO Box 4, Canberra Act 2600, Australia AULESTIA, V., Lang. Media Ctr-U of Mlaryland, 5401 Wilkens, Baltimore, MD 21228

AV LANGUAGE JOURNAL, 7 Shelley Close, Langley, bucks SL3 8JW, England AV LANGUAGE JOURNAL, Univ. Aston, Gosta Green, Birmingham B4 7ET, England BABIN, J., French Dept., St. Francis Xaxier Univ., Anligonish B2G 1CO, NS, Canada

BAKER, R., FL Supv-Educational Dept., 65S Fiont St., Columbus, OH 432:15

BAILEY, L., 48B Kresge, Northwestern U., Ij5y Sheridan Rd., Evanston, IL 60201

BALCAEN, H. L., Lang. Lab., UN Manituba, Winnipeg R3T 2M8, Man, Canada

BALL ST. UNIVERSITY, Library Service-Continuations, Muncic, IN 47306

BARNES, J., FL Supv-Dept. of Education, Agana, GUAM 96910

BARR, C., Language Lab., St. John's Seminary, 5118 E. Eeminary, Camarillo, CA 93010

BARTZ, W., Public Instruction, State House 229, Indianapolis, IN 46204

BAUMAN, R., Modern Languages, Pomona Cullege, Claremont, CA 91711

BEAUDOIN, J., Language Lab., Western Michigan University, Kalamazoo, MI 49008 
BENNINGTON COLLEGE, Language Lab., Bennington, VT 05201

BENT, A., 140 Brandeis Rd., Newton, MA 02159

BERNDT, R., Language Dept., Southwest Missouri State U., Springfield, MO 65802 BERROA-SANCHEZ, R., Lang. Lab., Wesleyan Univ., Fisk Hall, Middleton, CT 06457 BERRY, K., Media Srv., City College, 721 Cliff Dr., Santa Barbara, CA 93109 BEUSCH, A., Dept. of Education-Box 8717, BWL Airport, Baltimore, MD 21240 BIBLIOTHEEK, Rijksuniversiteit-Groningen, Jatstraat 5, Groningen, Netherlands BILINGUAL EDUCATION, P.O. Box 2219, Hartford, CT 06115

BORDIE, J., Language Educ. Library, $U$ of Texas, Austin, TX 78712 BOSWORTH, L., New Student Services, U of Wisconsin, Madison, WI 53706 BOUDREAU, J., Language Lab, Wheaton College, Norton, MA 02766 BOULWARE, R., AIMC, R. 1, Box 261, Fishersville, VA 22939

BOWEN LOUISE, 201 Bartlett, Dartmouth Coll., Canover, NH 03755 BOYDEN, P., 271 Library, Kent State University, Kent, OH $442+2$ BREVARD, COMM. COLLEGE, Receiving, 1519 Clearlake Rd., Cocoa, FL 32922 BRIDGERS, W., Phelps Hall-Lrng. Lab., U of 'California, Santa Barbara, CA 93106 BRITISH LIBRARY, Boston Spa-Wetherby, Yorkshire IS23 7BQ, England BRONX COMMUNITY COLL., Library-Learning Ctr., W. 181 St., Bronx, NY 10453 BROWN, C., Wilson Bldg 251, Western KY Univ., Bowling Green, KY 42101 BROWN UNIVERSITY, Language Lab Library, Box E., Providence, RI 02912 BUNKER, D., Languages, Appalachian State University, Boone, NC 28607 BURGGRAAF, S., Brigham Young University, W-162 Stadium, Provo, UT 84602 CALIFORNIA POLYTECH STATE U., Lib-Periodicals, San Luis Obispo, CA 93407 CALVIN COLLEGE LIBRARY, 3207 Burton Se., Grand Rapids, MI 49506 CAMPUSVE), TSS, SS, Univ-Bibliotek, DK-5230 Odense $M$, Denmark CAPRETZ, P., Language Lab, Yale University, 111 Grove St., New Haven CT 06510 CARLETON COLLEGE, Library, Northfield, MN 55057

CARLSON LIB-SERIALS, University of Toledo, Toledo, OH 43606 CATE, B., Box 260133, Tampa, FL 33685

CATE, R., Language Lab, University of Texas, El Paso, TX 79968 CELANDINE, T., Atlantic Community College, Mays Landing, NJ 08330 CHANC, G., Moore Hall 256, U. of Hawaii, Honolulu, HI 96822 CHEATHAM, R., Language Lab., U. of Arkansas, Little Rock, AR 72207 CHERCHIO, D. DE., FL Dept., West Chester State College, West Chester, PA 18380 CHERRY, C., Language Lab., Furman University, Greenville, SC 29613 CHERRY CREEK HIGH SCHOOL, 9300 E. Union Ave., Englewood, CO 80111 CHESTNUT, D., Educational Dept.-Box 911, Educational Bldg., Harrisburg, PA 17126 CICERAN, A. J., Lang. Lab., Brock Univ., St. Catharines 225 3A1, Ont., Canada CLAMEN, J., 88 Horner Ave., Toronto, M8Z 4X8, Ont., Canada 
CLARK, D., Lang. Lab.-11 Gilman, Johns Hopkins Univ., Baltimore, MD 21218

CLEVELAND STATE U., Library-Serials, 1860 E. 22nd St., Cleveland, OH 44115

COCHRAN, C., 205 N. Walnut St., Brazil, IN 47934

COFFEY, T. F., Language Lab., Creighton Univ., Omaha, NE 68178

COMET, H., Language Inst., 280 S. Beverly Dr., Beverly Hills, CA 90212

CONCORDIA UNIV., Lib.-Ser., 1455 Maisonneuve Blvd. W., Montreal H2G 1M8, PQ, Canada

CONWELL, M., 251 W. De. Kalb Pk, 909C, King of Prussia Pa., 19406

COLE, B., Spanish Language, University of Delaware, Newark, DE 19711

COLE, BENNETT, 207 Ardmore Blacksburg, VA 24060

COLL CAPE BRETON, Lib., Box 5300, Sydney B1P 6L2, NS, Canada

COLTHARP, J., Audio Visual, 340 Gaskill, Miami University, Oxford, OH 45056

CONNER, M., Central States Conference, U. of Nebraska, POB 688, Omaha, NE 68101 CORNETTE LIBRARY, West Texas State U., Box 748 Wt Station, Canyon, TX 79016 COUROUX, G., Col. M. Victorin, 7000 Rue M Victorin, Montreal, 462, PQ, Canada COX, S., Language Lab., Auburn University, Auburn, AL 36830

CREAMER, J., Language Lab., Hudson High School, Brigham St., Hudson, ME 01749 CRIMINALE, L., Language Lab., Elmira College, Elmira, NY 14901

CROSBIE, K., Bilingual Ed.-Publ. Instruction, Old Capitol Bldg., Olympia, WA 98504 CTR-INFORMATION LANGUAGES, 20 Carlton House Ter., London SW 1Y 5AP, England DALSTROM, E., U. of Nebraska, Foreign Languages, Omaha, NE 68112

DAMMER, P., Foreign Language Dept., Education Dept., Albany, NY 12234

LANGUAGE LAB, C. Dechaine, Univ. Alberta, Edmonton T6G 2E1, Alta, Canada

DENTE, E., Tufts University, 122 Eaton Hall, Medford, MA 02155

DIRECTOR, Dept. of Education, Bilingual Educ., Boston, MA 02111

DEJOHNSON, T. H., Mod. Lang., St. Louis Univ., St. Louis, MO 63103

DELACRUZ, J., Cranbrook, 380 Lone Pine Box 810, Bloomfield HLS, MI 48013

DE MERS 1., Harvard School, 3700 Coldwater Canyon, N. Hollywood, CA 91604 DLIWC ACADEMIC LIBRARY, Bldg. 618, Presidio, Monterey, CA 93940

DOLORES DE VARGAS, Inst. Idiomas, Casilla 51 Tarija, Bolivia

DODGE, J., Northeast Conference, Box 623, Middlebury, VT 05753

DONATH, T., Learning Ctr., Buena Vista College, Storm Lake, IA 50588

DOWLING, J., Language Lab, U. of Queensland, St. Lucia Brisbane 4067, Australia DUGAN, M., Learning Lab, Comm. Coll-Phila., 34 S 11 St., Philadelphia, PA 19107 DULA, EVA, For Lang. Lab., Delaware St. Coll., Dover, DE 19901

DUPER LIBRARY, U. of SW Louisiana, 302 E. St. Mary BIvd., La Fayette, LA 70504

DUKAS, V., Language Lab., San Diego State College, San Diego, CA 92182

DUVERLIE, CLAUDE, Mod. Languages-Linguistics, UMBC, Catonsville, MD 21228

EASTERN MONTANA COLLEGE, Library-Serials, Billings, MT 59101 
EARLE, B., Language Lab, 59 Henderson, Needham, MA 02192

EATON, S., 140 W. River St., Apt. E, Wilkes-Barre, PA 19040

ECONOMOS, R. M., Pace Univ., 41 Park Row, New York, NY 10038

EMDON LOW LIBRARY-SERIALS, OIkahoma State U., Stillwater, OK 74074

EK, JAMES, 16216 North 33rd Dr., Phoenix, AZ 85023

ELLISON- K., Languages-A \& S, West Georgia College, Caroliton, GA 30118

ELMENDORF, W., Language Lab., Western Washington St. Col., Bellingham, WA 98225

ENOS, I., Languages, Bridgewater St. College, Bridgewater, MA 02324

ENTIN, N., Gratz College, 10 St. \& Tabor Rd., Philadelphia, PA 19141

ERIC EXCHANGE LR, Ctr.-Linguistics, 3520 Prospect St. NW, Wash. D.C. 20007

FEAGIN, C., Dept. of Education, Education Annex-Trinity, Atlanta, G9 30303

FISHER, H., Visual Media, University of California, Davis, CA 95616

FISHER, R., Language Lab., La Salle Col., 20th \& Olney, Philadelphia, PA 19141

FORD, PENELOPE, Mod. Languages-Linguistics, UMBC, Catonsville, MD 21228

FOREICN LANGUAGES, Memphis State University, Memphis, TN 38152

FOURNIER, R., Bilingual Education, 64 N. Main-3rd Fl., Concord, NH 03301

FRANCIS, L., English Ctr., U. of Missouri, 229 Centry, Columbia, MO 65201

F RECHETTE, E., Languages, Hlorida State University, Tallahassee, FL 32306

FRITSCH, H., P.O. Box 61, Whately, MA 01093

FROEHLICH, J., Language Lab., Scripps College, Claremont, CA 91711

FROST, A., 304 E. Pyne, Princeton University, Princeton, NJ 08540

FUKUOKA UNIVERSITY, LL Rm., Nanakuma Fukuoka $814 \mathrm{MZ}$, Japan

GACE, A., FL Spec.-Educational Dept., Hodge Bldg.-N Lincoln, Oklahoma City, OK 73105

CAIKOKUCO, Nagoya Gakuin Daigaku, Kamishinano-Cho Seto Aichiken4, Japan

CALLOWAY, V., FL Consultant-Education Dept., 801 Futledge, Columbia, SC 29201

GARINGER, E., Univ. of Kansas, 4069 Wescoe Hall, Lawrence, KS 66045

GEMMER, B., Kamehameha Schools, Keona-Lower Campus, Honolulu, HI 96817

GeNNAULA, M. G., 5872 Kings Sch. Rd., Bethel Pk, PA 15102

GEORGIA INST. TECHNOLOCY, Library, 225 North Ave. NW, Atlanta, GA 30332

GERMAN QUARTERLY GERMAN \& SLAV, U. of Virginia, Charlottesville, VA 22901

GERMAN-SLAVIC LANCUACES, Texas Tech U., POB 4579, Lubbock, TX 79409

GIFU JYOSHI TANKI DAIGAKU, 2693 Fukumitsu, Nagara Gifu 502 MZ, Japan

GILDE, H., Oldfather IIII, University of Nebraska, Lincoln, NE. 68588

GIONET, A., Language Dept., North Texas State U., Denton, TX 76203

GOLDSMITH, S., Language Lab., 101B Phil. Hall, Columbia U., New York, NY 10027

COLDSWORTHY, T., 279 Van Hise, U. of Wisconsin, 1220 Linden, Madison, WI 53706

COODIN, J., Educatioal Media Div. Inc., Box 20604, Oklahoma City, OK 73156

GORDEN, D. L., Language Lab., Marquette Univ., Milwaukee, WI 53233

Spring/Summer 1980 
GORE, P., BOX 22, Wilmington, VT 05363

GORE, P., Box 11, Deerfield, MA 01342

GOULET, SR. N., Notre Dame College, Manchester, NH 03104

GOVERNORS STATE UNIVERSITY, Library-Kardex, Park Forest South, IL 60466

GREENBERG, E., 3099 Brighton St., Brooklyn, NY 11235

GRIFFITH, P., Education, 100 N. First, Springfield, IL 62777

GRITTNER, F., FL Education-Public Instructionn, 126 Langdon, Madison, WI 53702

GROSJEAN, C., Language Lab-84O DWinnelle, U. of California, Berkeley, CA 94720

GROSS, STEPHANIA, University Hall, Bowling Grn. State, Bowling Green, OH 43402

HALL, B. M., Monterey inst. Intl. St., 425 Van Buren, Monterey, CA 93940

HAMM, C., Language Lab, Queen's University, Kingston K7L 3N6, Ont, Canada HAMMERLY, H., Linguistics, Simon Fraser University, Burnaby V5A 186, BC, Canada HANZEL, L., Nanzan University, 18 Yamazato-Cho, Showa-Ku, Nagoya 466, Japan HARBOR CAMPUS, Media Ctr., Univ.-Massachusetts, Dorchester, MA 02125 HAROLD LEE LIBRARY-SERIALS, Brigham Young University, Provo, UT 84602 HARRIS, J., A-V Services, Stark Technical Coll., 6200 Frank NW, Canton, OH 44720 HARTWELL, 'J., Bowling Grn. State, Instr. 'Media Ctr., Bowling Green, OH 43403 HEATON, J. T., For. Lang., Colorado State U., Ft. Collins, CO 80523 HEILENMAN, L., Languages, Virginia Polytechnic Inst., Blacksburg, VA 24061 HELSINGIN YLIOPISTO, Fabianinkatu 26, SF-00170 Helsinki 17, Finland

HENNIG, C., Language Labll, ML Bldg., U. of Waterloo, Waterloo N2L 3C1, Ont, Canada

HERRERA, L., Westminster Schools, 1424 W. Paces Ferry N.W., Atlanta, GA 30327 HIROSHIMA DAI, Honbu Toshokan-K, Hiroshima 730 MZ, Japan HEWSON, J., Linguistics, Memorial University, St. John's Newfoundland, NF, Canada HILTS, M., La Sierra College?loma Linda U., Riverside, CA 92515 HIROSHIMA SHUDO DAIGAKU, Otsuka Numata-Cho, Hiroshima 731-31, Japan HIRSCH, B., Language Lab., Samford University, Birmington, AL 35209 HISPANIA, Editor, Romance Languages, U. of Cincinnati, Cincinnati, OH 45221 HOLMES, G., French Dept., U. of Western Ontario, London N6A 3K7, Ont., Canada HOLTON ARMS SCHL, Library, 7303 River Rd., Bethesda, MD 20034 HORWOOD LANGUAGE CTR., Univ. of Melbourne, Parkville Victoria, Australia HOWARD, J., Dept. of Education, 111 Coliseum Blvd., Montgomery, AL 36109 HOWARD, UNIVERSITY, Language Lab., 361 Lockehall, Washington, DC 20059 HOWARD UNIVERSITY, Library RM105-Serials, Washington, DC 20059 HOWE, E., FL Educ.-Education Dept., 250 E. 500 South, Salt Lake City, UT 84111 HUDSON, C., Dept. of Education, Little Rock, AR 72201 IKEURA, S., Fukuoka University-Educ., 279 Akama Munakata-Gun Fukuoka, Japan INFANTE, 1., Languages ,Public Instruction Dept., Raleigh, NC 27611 
INST. LANGUAGES, Bhar Bhasha Sansthan, Manasagangsti, Mysore 570 006, India INSTRUCTIONAL MATERIALS CTR., 201 E. Nine Mile Rd., Highland Sprgs, VA 23075 INTER-AMER UNIV., Library, San German, PR 00753

IRVING, E., Languages, Tennessee Tech University, Cookeville, TN 38501 JACKSON, D., Public Instruction, Second Languages, Helena, MT 59601 JACKSON, G., 257 Cliffview Dr., Gahanna, OH 43230

JACKSON, G., Language Lab., Capital University, Columbus, OH 43209 JARLETT, F., Language Dept., Ctl. Conn. St. College, New Britain, CT 06050 JEBE, S., Education-649 Capitol Sq., 550 Cedar St., St. Paul, MN 55101 JEWISH NATL.-UNIVERSITY LIBRARY, POB 503, Jerusalem, Israel JOHNSON, B., For. Language, Wash. St. Univ., Pullman, WA 99164 JOHNSTON, WANDA, 323 Candler Lib., Emory Univ., Atlanta, GA 30322 JORIA, G., Language Resource Ctr., Loyola University, Chicago, IL 60626 LANGUAGE LAB., Jyvaskyla University, SF-40-100 Jyashkla 10, Finland KAGAWA UNIV., Library, Saiwai-Cho, Takamatsu-Shi 760, Japan KAMEHAMEHA SCHOOLS, Kapalama Heights, Honolulu, HI 96817 KANSAI UNIV. LIBRARY, POB 50, Suita Osaka $564 \mathrm{MZ}$, Japan KATH, R., German Dept., University of lowa, lowa City, IA 52240 KATHOLIEKE UNIV. LEUVEN, Blijde Inkomststraat 21, 3000 Leuven, Belgium KENNEDY, D., Languages-Pr. George Schl., 4806 Harvard, College Park, MD 20740 KENNER, R., Land. Lab. AV, Concordia Univ., Montreal H3C 1M8, PQ, Canada KESSLER, M., Language Lab.-Humanities, U. of Houston, Houston, TX 77004 KILMER AREA LIBRARY, Rutgers Univ., San 351-0158, New Brunswick, NJ 08903 KINC, R., Education Dept., P.O. Box 480, Jefferson City, MO 65102 KIPPHORN, R., Language Lab., St. Joseph's Univ., Philadelphia, PA 19131 KIRK, D., 124 Chace Street, Santa Cruz, CA 95060 KITAO, K., Intl. Ctr., 39-1 Koyama Shimofusa Cho-Kita, Kyoto 603, Japan KODAMA, P., Lrng. Assistance Ctr., Hawaii Pacific, 1164 Bishop, Honolulu, HI 96813 KOESTER, A., Dept. of Education, Frankford, KY 40601 KOHMOTO, S., 4-6-17 Meguro, Meguro-Ku, Tokyo 153, Japan KOLTAY, E., R. R. Bowker Co., 2 Stewart Ave., Eastchester, NY 10707 KONWTAR, E., Pob 483, Budapest 1372, Hungary KORKEAKOULUN, J., Kirjasto PI. 111, 80101 Joensuu 10, Finland KOTO, K., Senmon Kakko Library, Aga-Minami-2-2-11 Gure 737 MZ, Japan KOZORIZ, G., Language School, NIt. Defense Hdqs., Ottawa KIA OK2, Ont, Canada KUMP, R., 1822 Ricardo Dr., Cape Ciradreau, MO 63701 KURITA, K., Sony Lab.-Hiyama Bldg. \#2, 4FL, 2-20-30 Takanawa, Tokyo 108, Japan KRUSE, E., 733 Kirkwood De., Grand island, NY 14072 KURODA, T., 2477 Kugenuma, Fujisawa Clty 251, Japan 
KYOTO SANGYO DAIGAKU, Toshokan, Kita-Ku Kyoto $603 \mathrm{MZ}$, Japan

KYUSHU SANGYO DAIGAKU, 327 Matugadai-2-Chome, Fukuoka $813 \mathrm{MZ}$, Japan

LACHER, S., Languages-Public Instruction, Capital Bldg., Bismarck, ND 58505

INTL STUDIES, N Lacharite, St. Michael's College, Winooski, VT 05404

LALANDE, J., 1229 S. Allen St. \#29, State College, PA 16801

LALLY, D. V., Learning Lab., U. of Louisville, Louisville, KY 40292

LAMERAND, R., 252 Bloor St. West, Toronto M5S 1V6, Ont, Canada

LAMORE, M., Language Lab., Cottey College, Nevada, MO 64772

LANGE, D., U. of Minnesota-130B Peik., 159 Pillsbury Dr. Se., Minneapolis, MN 55455 LANGUAGE LABORATORY C-088, Univ. of California-San Diego, La Jolla, CA 92093 LANGUAGE \& LANGUAGe BeHAVIOR, Abstracts, POB 22206, San Diego, CA 92122 LAROCHE, J., Lang.-D. C. Public Schools, 415 12th St., N.W., Washington, DC 20004 LAWRASON, R., Media Lrng. Ctr., Temple University, Philadelphia, PA 19122 BUCHHANDLUNG LEHMKUHL, Leopoldstr 45, 8000 Munchen 40, West Germany LEMYZE, J-C, Language Lab., McGill University, Montreal H3A 1X9, PQ, Canada LEVINSON, B., Modern Languages-Lab., Brooklyn Col.-Cuny, Brooklyn, NY 11210 LHTEC, 905 Lindsey Hopkins Library, 1410 NE 2nd. Ave., Miami, FL 33132 LIBRARY OF CONGRESS-ORDER DIV., Section C, Washington, DC 20540 LIDJI, M., Language Lab.-Cuny., 133 \& Convent Ave., New York, NY 10031 LINGUISTICS INST. BELGIUM, Blijde Inkomststraat 21, 3000 Leuven, Belgium LONG BEACH CITY COLL., Foreign Lang., 4901 E. Carson, Long Beach, CA 90808 LORE, A. G., Romance Languages-Dey. Hall., Univ. NC, Chapel Hill, NC 27614 LUTZ, DAVID, 340 Pershing Drive, Las Vegas, NV 89107 MACINKO, J., Language Lab., U. of Colorado, 302 Old Main, Boulder, CO 80309 MACKEY, C. R., For. Lang., Simmons Coll., Boston, MA 02115 MAGILL, R., Language Lab, Towson State University, Baltimore, MD 21204 MAGIN, J. F., Univ. Western Ontario, London 72, Ont., Canada MANTINI, B. K., Languages, N Hennepin Comm. Coll., Minneapolis, MN 55445 MARIETTA COLLEGE, Modern Language Dept., Marietta, OH 45760 MARINO, A., Language Lab., Hunter College, 695 Park Ave., New York, NY 10021 MARXHEIMER, E., Romance Lang., Univ. Alberta, Edmonton T6C 2E1, Alta, Canada U OF MARYLAND, Library-Serials, 5401 Wilkens, Baltimore, MD 21228 MASON, R., Humanities, Michigan Tech. University, Houghton, MI 49931 MATTHIES, B., English Dept., lowa State University, Ames, IA 50011 MAZZEO, C., 441 Monroe Hall, George Washington Univ., Washington, DC 20052 MAYNES, J., Dept. of Education, 1535 W. Jefferson, Phoenix, AZ 85007 MEDGAR EVERS COLLEGE, Cuny, 1127 Carroll St., Brooklyn, NY 11225 MEMIN, J., Rayzor Hall 106, Rice University, Houston, TX 77005 MESSNER, C., Languages, Carlton College,, Northfield, MN 55057 
METCALFE, J., Language Laboratory, Stanford University, Stanford, CA 94305

MEYER, M., Language Lab., Hamilton College, Clinton, NY 13323

MILLER, J., Wittenberg University, Springfield, OH 45501

MILLER, W., 310 Olin Hall, University of Akron, Akron, OH 44325

MILLS, M., Comm. Lab., Brookhaven Col., 3939 Valley View Ln., Farmers Br., TX 75234

MINISTRY OF CULTURE, Lang. Class, 454 University Ave, Toronto M4G 1Y7, Ont., Canada

MINISTRY OF CULTURE, Resource Ctr., 77 Bloor St. W., Toronto M7A 2R9, Ont, Canada SHOGO MIURA, Hiroshima U., 1-1-89 Higashi-Senda-Machi, Hiroshima 730, Japan

ADV. MGR. MLA, 62 Fifth Avenue, New York, NY 10011

MOE, P., Tandberg, Inc., Labriola Court, Armonk, NY 10504

EDITOR, MODERN LANGUAGES JOURNAL, 314 Cunz, Ohio State U.,. Columus, OH 43210

MOLDIN, C., 6501 Lowland Dr., Landover, MD 20786

MONASH UNIVERSITY, Periodicals, Clayton Victoria 3168, Australia

MOOREHEAD STATE UNIVERSITY, Library-Serials, Moorehead, MN 56560

MORRISON, R., So. Missionary College, Box 475, Collegedale, TN 37315

MOSEBY, E., Tandberg A-S, P.O. Box 55-Bogerud, Osla 6, Norway

MOSES, J., Stephen F. Austin State U., Box 3042, Nacogdoches, TX 75962

MURRAY, W., Els. Sound Production, 14731 Oxnard St., Van Nuys, CA 91411

MCCARTY, J., Hofstra Univ., Language Lab.-106 Calkins Hall, Hempstead, NY 11550

MCCUNE, J., Language Lab., Indiana U-Ballantine 120, Bloomington, IN 47401

MCGILL UNIV. LIB-SER., 3459 Mctavish, Montreal H3A 1Y1, PQ, Canada

MCKAY, J., Speech Communication, University of Texas, Austin, TX 78712

MCNAB, J., Languages, Virginia Polytechnic Inst., Blacksburg, VA 24061

MCNEAL, G., Nursing, University of Pittsburgh, Bradford, PA 16701

NANYANG UNIV., Library, Upper Jurong Rd., Singapore 22, Singapore

NASH, D., Roberts Wesleyan College, North Chili, NY 14514

NASSAU COMM. COLLEGE, Library, Stewart Ave., Garden City, NY 11530

NATIONAL INSTITUTE OF EDUCATION, Educa Research Lib., Washington, DC 20208 NATL LIBRARY-AUSTRALIA, NS 110-72, Canberra Act 2600, Australia

NATL. LIB. CANADA, Order Sect. 395 Wellington St., Ottawa K1A ON4, Ont, Canada NEILSEN, M., Education Dept., 301 Centennial Mall S, Lincoln, NE 68509

NELSON, R., Col. Dept., John Wiley \& Sons, 605 Third Ave., New York, NY 10016

NICHOLSON, C., Lyons 313, Boston College, Ci.estnut Hill, MA 02167

NICHOLSON, C., Dept. of Education, 120 E. 10th, Topeka, KS 66612

NIEMAN, L., 11 Gary PI., Wappingers Falls, NY 12590

NORRISH, N., Lang. Lab., University of Victoria, Private Bag., Wellington, New Zealand NORTH TEXAS STATE U., Acquisitions, POB 5188 NT Station, Denton, TX 76203

NUFFER, J., Lrng. Lab., Portland State University, POB 751, Portland, OR 97207

Spring/Summer 1980 
NUIBE, Y., Fukuyama Univ., Higashimura-Machi, Fukuyama 729-02 MZ, Japan O'BRIEN, P., 280 Richmond Avenue, Amityville, NY 11701

O'HEARON, SR, FI. Lab., Col-White Plains, 78 N. Broadway, White Plains, NY 10603 OCHOA, A., Dept. of Education, 650 W. State-Jordan Bldg., Boise, ID 83720 OHIO STATE U., Library-Serials, 1858 Neil Ave., Columbus, OH 43210 OLPHEN, V., Language Lab., Batts Hall 1, U. of Texas, Austin, TX 78712 OLSEN, I., 5 Moellering Library, Valparaiso U., Valparaiso, IN 46383 ORT, B., Dept. of Education, P.O. Box 30008, Lansing, MI 48909 OUELLETTE, R., GRP1 Bldg.-207, So. Massachusetts Univ., N. Dartmouth, MA 02747 PARR, S., Language Lab., Juniata College, Huntingdon, PA 16652 PATRON, G. A., Language Lab., Univ. New Orleans, New Orleans, LA 70122 PENN STATE LIBRARY, Serials, Box 1601 Rm. 46, Harrisburg, PA 17126 PENSACOLA JUNIOR COLLEGE, Library, 1000 College Blvd., Pensacolal 32504 PENTA, R., Belmont School System, 38 Normandy Rd., Lexington, MA 02173 PERDUE, L., FI. Lab., Oklahoma State University, Box 1204, Stillwater, OK 74074 PETERSON, G., Foreign Languages, University of Nevada, Reno, NV 89507 PERIODICALS SRV. CTR., Hill Library-Box 5007, N. C. State, Raleigh, NC 27650 PETERSON, G., Notre Dame Womens College, Shimogamo, Sakyo-Ko Kyoto 606, Japan PETHERBRIDGE, D., University of Lethbridge, Lethbridge T1K 3M4, Alta, Canada PFLANZ, B., Dept. of German, University of Redlands, Redlands, CA 92373 PIERCE, J., English Dept., Del Mar College-East, Corpus Christi, TX 78404 PINEYRO, R., Dept. of Education, 721 Capitol Mall, Sacramento, CA 95814 PLEASANTS, J., 20 Dewey Lane, Hampton Bays, NY 11946 POHLMEIER, R., Educational Media Div., Inc., Box 20604, Oklahoma City, OK 73156 POLLY, L., Language Dept., Southwest Missouri State, Springfield, MO 65802 POPE, D., Language Lab., University of the Pacific, Stockton, CA 95211 PORTILL, M., Manhattanville College, Purchase, NY 10577 POWELL, R., Learning Ctr., Andrews University, Berrien Springs, MI 49104 PULLLN LIBRARY-PERIODICALS, Bowie State College, Bowie, MD 20715 PURDUE UNIVERSITY LIB., PCF Kardex, West La Fayette, IN 47907 QUEENS COLLEGE, Library-Serials, P.O. Box 6702, flushing, NY 11367 RAMSAY, P., Language Lab., Eastern Michigan University, Ypsilanti, MI 48197 RAPINCHUK, G., Missouri Western St. Col.-Lrng. C, 4525 Downs, St. Joseph, MO 64507 RAWLINGS, R., 101 Kobuke Cho, Chiba Shi Chiba Ken Z81, Japan REEVES, G., Language Lab, Univ. of Newcastle 2308, Newcastle NSW, Australia REIMERS, THERESIA E., Mod. Lang., Hollins College, VA 24020 RENECAR, C., Language Lab., Occidentau College, Los Angeles, CA 90041 RICHARDSON, MARTHA, Boylston G-3, Harvard, Cambridge, MA 02138 RICHMOND, E., Modern Languages, GA Inst. of Technology, Atlanta, GA 30332 
RIEKEHOF, L., Sign Language, Gallaudet Coll., Kendall Grn., Washington, DC 20002 RIVERS, W., 207 Boylston, Harvard University, Cambridge, MA 02138 RODEWALD, J., Language Lab., Central Mich. University, Mt. Pleasant, MI 48858 ROESCH LIBRARY-Periodicals, University of Dayton, Dayton, OH 45469

ROGERS, J., Modern Languages, Lincoln University, Lincoln, PA 19352

ROLLIN, M., 640 Wormwood Hill Rd., Storrs, CT 06268

ROWE, G., Foreign Languages, University of Idaho, Moscow, ID 83843

ROYALTY, J., Language Media Ctr., Univ. of Maryland, College Park, MD 20742

RUSDEN STATE COLLEGE, Sarjeant Library, Clayton Victoria 3168, Australia

SACA UNIV., Honjyo-Machi, Saga City $840 \mathrm{MZ}$, Japan

SAILORS, R. E., Edgecliff Alcohol Ctr., S. 511 Park Rd., Spokane, WA 99216

SAN DIEGO STATE UNIV., Love Library-Serials, San Diego, CA 92182

SAWYER, I., Languages, Simon Fraser Univ., Burnaby, BC, Canada

SAYLOR, L., Language Lab., Box 413, U. of Wisconsin, Milwaukee, WI 53201

SCHAEDEL, G. E., Kay Elemetrics, 12 Maple Ave., Pinebrook, NJ 07058

SCHLADWEILER, K., Language Arts-Education Div., Kneip Bldg., Pierre, SD 57501

SCHOBEL, D., 7801 Sagamore Dr., Cincinnati, OH 45236

SCHULTZ, D., Media Serv., Lehman Coll., Bedford Pk. Blvd. West, Bronx, NY 10468

SCHWARTZ, L., Language Lab., Emory University-402 Humanitie, Atlanta, GA 30322

SCLAFANI, C., Language Lab., Westchester Comm. Coll., Valhalla, NY 10595

SEA ENTERPRISES, 292-15-16 Luk. Luang. Rd., Bangkok 3, Thailand

SEAMEO LANGUAGE CTR., 30 Orange Grove Rd., Singapore 10, Singapore

SEIDEL, J., Language Lab., U. of Victoria, Box 1700, Victoria V8W 2Y2, BC, Canada SEINAN GAKUIN UNIV., Lang. Lab., 6-2-92 Nishijin Nishi-Ku, Fukuoka 814, Japan

SELLARS, S., Foreign Languages, University of Arkansas, Little Rock, AR 72204

SENC, M., University of Texas, 2505 Princeton Dr., Austin, TX 78741

SERAfINO, R., Languages, New Haven Pub. Schools, POB 3816, New Haven, CT 06525

SERGIO RIOS JABRYQUINTO, Casilla 165, Tarija, Bolivia

SEYDOUX, A., Language Res. Ctr., Loyola Univ., 7101 W. 89th St., Los Angeles, CA 90045

SHEEHAN, J. H., Language \& Culture-English, Univ. Houston, Houston, TX 77004

SIMON FRASER UNIVERSITY, Library, Acquisitions, Burnaby V5A 156, BC, Canada

SLAVENS, M., Languages, Rockford College, Rockíord, IL 61101

SMITH, K., Drop-In Lab., New Mexico State U., Box 5278, Las Cruces, NM 88003

SMITH, E., FL Spec-Education Dept., Memphis St. University, Memphis, TN 38152

SMITH, P., Languages, West Chester State College, West Chester, PA 19380

SMITH, W., Languages, Purdue University, La Fayette, IN 47907

SMITHER, W., Spanish \& Portuguese, Tulant Univ., New Orleans, LA 70118

SO ILLINOIS UNIVERSITY, Library-Periodicals, Carbondale, IL 62901

Spring/Summer 1980 
SOMMER, U., Friendship Library, Fairleigh Dickinson U., Madison, NJ 07940

SOUMOKIL, P., Bilingual Education, Hathaway Bldg., Cheyenne, WY 82002

SPINKS, O., Humanities B-16, Suny, Albany, NY 12222

SR HIGH SCHOOL, Languages, 50 Rice St., Wellesley Hills, MA 02181

ST. UNIV. BIBL. FRA 011, Bockenheimer Landstr 134-138, 6 Frankfurt Main, West Germany

ST. UNIV. COLLEGE, Butler Lib.-Box P, 1300 Elmwood Ave., Buffalo, NY 14222

STALEY, H., Education Dept., P.O. Box 771, Jackson, MS 39205

STANFORD UNIVERSITY, Library-Serials, Stanford, CA 94305

STEELE, K. L., Ori-Rm. 1012 GPA, Univ. Florida, Gainesville, FL 32611

STERN, R., 201 East 17 Street, New York, NY 10003

STIEHL, R., School of Education, Oregon State University, Corvallis, OR 97331

STRAUBER, I., Telex Communications, Box 182, Encino, CA 91316

STROZIER LIBRARY-SERIALS, Florida State University, Tallahassee, FL 32306

SUNNY, Library Vestal Parkway East, Binghamton, NY 13901

SUPV.-LANGUAGES, Education Dept., P.O. Box 630, St. Thomas, VI 00801

SWANSON, S., Language Lab., University of Wisconsin, Oshkosh, WI 54091

TAKACX, L., Administration Center, 5933 West 115th St., Worth, IL 60482

TANNER, J., Language Lab., U. of Virginia, Charlottesville, VA 22903

TAYLOR, W., Spanish Dept., Upsala College, East Orange, NJ 07019

TEACHERS COLleGe LIBRARY, Serials, Box 307, 525 W. 120 St., New York, NY 10027

TEXAS EDUC. AGENCY, Resource Ctr. Lib., 201 E. 11th St., Austin, TX 78701

THIBAULT, CLAUDE, Notre-Dame-De-Foy, Cap Rouge GOA 1KO, PQ, Canada

THOMAS, J. E., Learning Lab., Virginia Polytechnic Inst., Blacksburg, VA 24061

TOKYO-SEITOKU-TANKI-DAIGAKU, 7-13 Jujodai-1-Chome, Kita-Ku, Tokyo, Japan

TOMPINS, E., Forsyth Tech. Ctr. Inst., 2100 Siles Cr. Pkw., Winston-Salem, NC 27103

TORRE, E. DE, Queens College-Cuny., Flushing, NY 11367

TRACY, R., Language Lab., Ridgewood, HS, Ridgewood, NJ 07450

TROMETER, R., M.I.T., Language Lab.-Bldg. 14N-0641, Cambridge, MA 02139

TURNER, J., Language Lab., Mercer University, Macon, GA 31207

UNIV. BERN-VERWALTUNC, Hochaschulstr 4, 3012 Bern, Switzerland

UNIV. BIBL., Zeitschriften Fgsz, Universitatsstrasse 4, 8520 Erlangen, West Germany

UNIV. BIBLIO 1439, A 324 639, Eras Muslaan 36, Nigmegen 1, Netherlands

UNION COLLEGE, Library, 3800 S. 48th St., Lincoin, NE 68506

UNIV. COLORADO, Library-Serials, Boulder, CO 80309

UNIV. CONCEPCION, Biblioteca, Casilla 1807, Concepcion, Chile

UNIV. DESTRASBOURG, Inst. Linguistique, 22 Rue Descartes, Strasboug 67084, France

UNIVERSITY OF GEORGIA, Library-Periodicals, Athens, GA 30602

UNIVERSITY O FILLINOIS, Language Learning Lab., Urbana, IL 61801 
UNIV. KANSAS LIBRARIES, Periodicals-Serials, Lawrence, KS 66045

U. OF KENTUCKY, Language Lab., 312 Classroom Bldg., Lexington, KY 40506 UNIV. LETHBRIDCE, Library, Lethbridge T1K 3M4, Alta, Canada

U. OF LOUISVILLE, Library-Serials, Attn: Mrs. Spradling, Louisville, KY 40292 UNIVERSITY OF MINNESOTA( Libraries-Serials, Minneapolis, MN 55445 UNIVERSITY OF MISSOURI, Library-Serials, Columbia, MO 65201 UNIV. NEBRASKA, Eppley Library-Periodicals, 60th \& Dodge, Omaha, NE 68182 U. OF NEBRASKA, Library-Periodicals, Omaha, NE 68182 UNIV. NEUCHATEL, Chaire Linguistique, Neufchatel 2000, Switzerland UNIV. NEWCASTLE, Auchmuty ibrary-Serials, Newcastle NSW 2308, Australia UNIV. NEVADA, Library-Periodicals, Las Vegas, NV 89154 NEVADA, UNIV., Library-Serials, Reno, NV 89507 U. OF RHODESIA, Language Lab., Box MP 167, Mt. Pleasant, Salisbury, Zimbabwe USAF ACADEMY, Library Dfslbs, Colorado Springs, CO 80840 UNIV. S AFRICA, Library, Private Bag 6058, Port Elizabeth 6000, Union S. Africa U. ST. ANDREWS, Language Library, St. Andrews, FIFE KY16 9PH, Scotland UNIV. STELLENBOSCH, Library, Private Bag 5036, Stellenbosch, Union S. Africa UNIV. SYDNEY, Lib.-Serials, Sdyney NSW 2006, Australia UNIV. OF TENNESSEE, Library-Serials, Knoxville, TN 37916 UNIVERSITY OF TURKU, Language Ctr., 20500 Turku 50, Finland UMMEL, C., Valley View Hs., 6027 Farmersville Pk., Germantown, OH 45327 UNIV. WEST ONTARIO, Lib.-Period, London N6A 3K7, Ont., Canada

U. WISCONSIN, Library-Serials, 728 State St., Madison; WI 53706

U. OF WISCONSIN-MILWAUKEE LIBR., P.O. Box 604, Milwaukee, WI 53201 U. WISCONSIN-PARKSIDE, Library-Serials, Wood Road, Kenosha, WI 53141 VALESKA, M., English Dept., Colorado State U., Fort Collins, CO 80523 VANN, S., Language Lab., Georgetown Univ., Washington, DC 20057 VERNICK, J., Language Lab-732 C of L, Univ. of Pittsburgh, PA 15260 VIJAYAKUMAR, E., Thurber Hs., 15 Orilla Park, Red Deer T4N 5A6, Alta Canada VIRCINIA POLYTECHNIC INST., Newman Library-Serials, Blacksburg, VA 24061 WAGUESPACK, P., Language Educ., Box 44064, Baton Rouge, LA 70804 WARKENTIN, H., Language Dept., Shippensburg State, Shippensburg, PA 17257 WARRINER, H., Bilingual Education, P.O. Box 6Q, Richmond, VA 23216 WATSON, J., Language Lab., Virginia Union University, Richmond, VA 23220 WAYNE COU. SCHOOL DISTRICT, MS B Katz, 33400 Van Born Rd., Wayne, MI 48184 WEIBLE, D., Audio Information, U. of Illinois, Box 4348, Chicago, IL 60681 WEINKAUF, A., Languages, Michigan Tech. University, Houghton, MI 49931 WELLS, S., Els. Languages Ctr., 1666 Cross Center Dr., Norman, OK 73069 WESSEL, Tandberg, Inc., Labriola Court, Armonk, NY 10504 
WEST CHESTER STATE COLLEGE, Library, West Chester, PA 19380

WHEATON COLLEGE, Norton, MA 02766

WIESE, P., Language Lab., Southern Conn. College, New Haven, CT 06515

WILDE, K., 1605 N. Fillmore St., Arlington, VA 22201

WiLLECKE, F., Foreign Languages, Wagner College, Staten Island, NY 10301

WILLIAMS, C., Language Lab., State University, Oswego, NY 13126

WILLIAMS-SONY, K. M., 411 Gordon Baker Rd., Willowdale M2H 256, Ont., Canada

WILl.lamsON, M., Lang. Lab., Notre Dame Acad, 30 Church St., Waterbury, CT 06702

WILLIS, R., 829 Shimkin Hall, 50 West 4th St., New York, NY 10003

WILLISTON, MRS. J., Lang. Lab.-Conn. Coll., Box 1524, New London, CT 06320

WILTON, HICH SCHOOL, Library, 395 Danbury Rd., Wilton, CT 06897

WIPF, J., 121 East Qnox Dr., West La Fayette, IN 47906

WOLLSTEIN, J., Dept. Ed.-For. Lang., 1270 Queen Emma, Honolulu, HI 96813

WOOD, R., Instructional Media, Utah State University, Logan, UT 84322

WOOLLEY, R., Instructional Media, Utah State University, Logan, UT 84322

WYATT, O., English-American Lang., Academy, Catholic Univ., Wash., DC 20064

WYNN, W., 975 Ventura Ave., Albany, CA 94707

YELLOW TEACHER'S SEMINARY, Beth Hakerm, Jerusalem, Israel

YIN, P., Media Lab., Cleveland State Univ., Cleveland, OH 44115

YORK UNIV. LIBRARIES, 4700 Keele St., Downsview M3) 2R2, Ont., Canada

ZAETTA, R., Dept. of Instruction, Townsend BIdg., Dover, DE 19901

ZIMMERMAN, MRS. M., Language Lab., Amherst College, Amherst, MA 01002

ZINGIS, A., Language Lab.-Old Crem. Bldg., U. of Cincinnati, Cincinnati, OH 45221 